\title{
Single ZnO Nanowire-Based Gas Sensors to Detect Low Concentrations of Hydrogen
}

\author{
Marlene N. Cardoza-Contreras 1,*, José M. Romo-Herrera 1,t, Luis A. Ríos ${ }^{2,+}$, \\ R. García-Gutiérrez ${ }^{3, \dagger}$, T. A. Zepeda ${ }^{1, \dagger}$, Oscar E. Contreras ${ }^{1}$ \\ Received: 28 October 2015; Accepted: 1 December 2015; Published: 4 December 2015 \\ Academic Editor: Michael Tiemann \\ 1 Centro de Nanociencias y Nanotecnología, Universidad Nacional Autónoma de México, \\ Baja California 22800, Mexico; jmromo@cnyn.unam.mx (J.M.R.-H.); trino@cnyn.unam.mx (T.A.Z.); \\ edel@cnyn.unam.mx, ocontrer@yahoo.com (O.E.C.) \\ 2 Centro de Investigación Científica y de Educación Superior de Ensenada, Ensenada, Baja California 22860, \\ Mexico; 1rios@cicese.mx \\ 3 Departamento de Investigación en Física, Universidad de Sonora, Hermosillo 83000, Mexico; \\ rgarcia@cifus.uson.mx \\ * Correspondence: marlenenohemi@hotmail.com; Tel.: +52-646-127-3968 \\ + These authors contributed equally to this work.
}

\begin{abstract}
Low concentrations of hazardous gases are difficult to detect with common gas sensors. Using semiconductor nanostructures as a sensor element is an alternative. Single $\mathrm{ZnO}$ nanowire gas sensor devices were fabricated by manipulation and connection of a single nanowire into a four-electrode aluminum probe in situ in a dual-beam scanning electron microscope-focused ion beam with a manipulator and a gas injection system in/column. The electrical response of the manufactured devices shows response times up to $29 \mathrm{~s}$ for a $121 \mathrm{ppm}$ of $\mathrm{H}_{2}$ pulse, with a variation in the nanowire resistance appreciable at room temperature and at $373.15 \mathrm{~K}$ of approximately $8 \%$ and $14 \%$ respectively, showing that $\mathrm{ZnO}$ nanowires are good candidates to detect low concentrations of $\mathrm{H}_{2}$.
\end{abstract}

Keywords: nanowire; sensors; hydrogen; SEM-FIB; nano-fabrication; devices.

\section{Introduction}

Conductometric gas sensors are electronic devices with a simple structure where their operation principle is based on the variation of electrical conductance of their sensor element; this can occur due to the electron exchange between the surface and conduction band as the result of oxidation/reduction when its surface is exposed by chemical gases. Semiconductors are one of the most commonly used materials for sensor elements, since naturally, in ambient air, a thin layer of native oxide is formed on a semiconductor's surface, and in the presence of a reducing gas, the interaction of oxygen-adsorbed species on its surface and the objective gas molecules causes the chemical reduction of its surface. This phenomenon modifies the amount of active charge carriers in the semiconductor and, hence, its conductance.

Most conventional gas sensors are fabricated with microscopic sensor elements, which are unable to detect very low concentrations (parts per million, pmm) of gases. Nowadays, mandatory safety regulations for handling harmful and hazardous chemicals for human beings and our surrounding environments strictly demand faster and highly sensitive sensors.

Semiconductor nanostructures as wires offer a great alternative to this problem, mainly due to their limited size and cylindrical geometry. $\mathrm{ZnO}$ is an n-type semiconductor, and when $\mathrm{O}_{2}$ molecules are adsorbed on a $\mathrm{ZnO}$ nanowire surface, they extract electrons from the conduction band and trap them at the semiconductor surface. This will lead to an electron depletion layer, and then there is no 
conduction on the semiconductor surface, and the remaining free charge carriers are concentrated in the bulk of the nanowire (conduction channel). Under the presence of a reducing gas, the depletion layer disappears due to the release of the electrons back into the nanowire, thus expanding the conduction channel, resulting in an increment of the nanowire conductance. The conduction in nanowires greatly depends on the density of trapped charges on their surface or the release of these charges back into the nanostructure. Specifically, $\mathrm{ZnO}$ has attracted considerable interest in electronic and photo-electronic devices such as UV/visible photodetectors and active components in solar cells and gas sensors [1-6]. ZnO is a direct wide-band-gap semiconductor (3.37 eV at RT), naturally grown as an n-type semiconductor, that according to the literature has an intrinsic behavior that results from intrinsic crystal defects such as oxygen vacancies and/or zinc interstitials. Materials with this feature are good candidates in the fabrication of fast-response and highly sensitive gas sensor devices for reducing atmosphere when n-type nanostructures are used as sensor elements.

The sensitivity of gas sensors can be highly dependent on temperature. Tien et al. [7] analyzed the hydrogen-sensing of multiple $\mathrm{ZnO}$ nanorods with Pt coating capable of detecting $500 \mathrm{ppm}$ of hydrogen at room temperature. A higher temperature was used by Tien et al. in single-crystal $\mathrm{ZnO}$ nanowires coated with $\mathrm{SnO}_{2}$ showing a strong sensitivity to $500 \mathrm{ppm}$ of $\mathrm{H}_{2}$ in $\mathrm{N}_{2}$ at $673.15 \mathrm{~K}$ [8].

Recently, Ranwa et al. [9] fabricated a Au/ZnO NRs/Si/ZnO NRs/Au Schottky junction-based nanosensor at different temperatures with $1 \%$ and $5 \%$ hydrogen environment and results showed that the sensitivity increases from $11 \%$ to $67 \%$ with temperature increasing from $323.15 \mathrm{~K}$ to $423.15 \mathrm{~K}$, and they also showed a response of $14 \mathrm{~s}$ for $1 \%$ at an operating temperature of $373.15 \mathrm{~K}$.

In this work we studied the conductometric response (electrical resistance) of single $\mathrm{ZnO}$ nanowires tested as gas sensor elements in order to explore their capability to detect very low concentrations of $\mathrm{H}_{2}$ at room temperature and at $373.15 \mathrm{~K}$.

\section{Experimental Section}

Gas sensor devices were fabricated using $\mathrm{ZnO}$ nanowires electrically connected to a four-electrode aluminum ( $\mathrm{Al}$ ) probe. A typical photolithography process was applied to $\mathrm{Si} / \mathrm{SiO} x / \mathrm{Al}$ substrates to produce the patterned $\mathrm{Al}$ probes. $\mathrm{Si} / \mathrm{SiO} x / \mathrm{Al}$ layer structures were grown by a deposition of an $\mathrm{Al}$ thin layer on $\mathrm{SiO}_{\mathrm{x}}$ thermally grown on silicon ( $\mathrm{Si}$ ) wafers. The $\mathrm{ZnO}$ nanowires were synthesized on $\mathrm{Si}$ substrates by chemical vapor deposition as described previously by Garcia-Gutierrez et al. [10]. The electrical connection process of each single nanowire over the Al probes was carried out in-situ in a dual-beam SEM-FIB (Jeol JIB-4500) equipped with a gas injection system (GIS) to perform Pt deposits and an Omniprobe Autoprobe 200.2 manipulator. Three single $\mathrm{ZnO}$ nanowire gas devices were fabricated using nanowires with diameters of 800,700 and $500 \mathrm{~nm}$, which are labeled $\mathrm{M}_{1}, \mathrm{M}_{2}$ and $\mathrm{M}_{3}$, respectively. I-V curves for all devices were acquired by electrical measurements carried out with an ECOPIA HMS-5000 Measurement System under room conditions, atmospheric-pressure and temperature conditions. Sensor devices were tested in a homemade gas chamber capable of performing time-dependent resistance measurements under well-controlled $\mathrm{H}_{2}$ atmospheres and temperatures up to $423.15 \mathrm{~K}$. Very low $\mathrm{H}_{2}$ concentration atmospheres were achieved by carefully mixing dry air and $1 \% \mathrm{H}_{2}$-argon balanced gases.

Later, gas sensor devices were introduced into the homemade gas chamber to evaluate their performance for detecting low $\mathrm{H}_{2}$ concentrations. Electrical resistance was now temporally monitored as a function of $\mathrm{H}_{2}$ concentration inside the chamber. The performance experiments are carried out using a continuous flow of dry air as a gas reference with a short pulse of a well-controlled $\mathrm{H}_{2}$ concentration.

Response is considered as the resistance variation percentage and was calculated through the next equation:

$$
\Delta R=\left[\frac{\left(R_{a}-R_{g}\right)}{R_{a}}\right] 100
$$

where $R_{a}$ and $R_{g}$ are the $\mathrm{ZnO}$ nanowire resistances before and during the $\mathrm{H}_{2}$ pulse, respectively. 


\section{Results and Discussion}

Figure 1 shows a SEM image of the fabricated $\mathrm{M}_{1}$ gas sensor device displaying the $\mathrm{ZnO}$ nanowire connected to the $\mathrm{Al}$ probe through Pt strips deposited by the GIS.

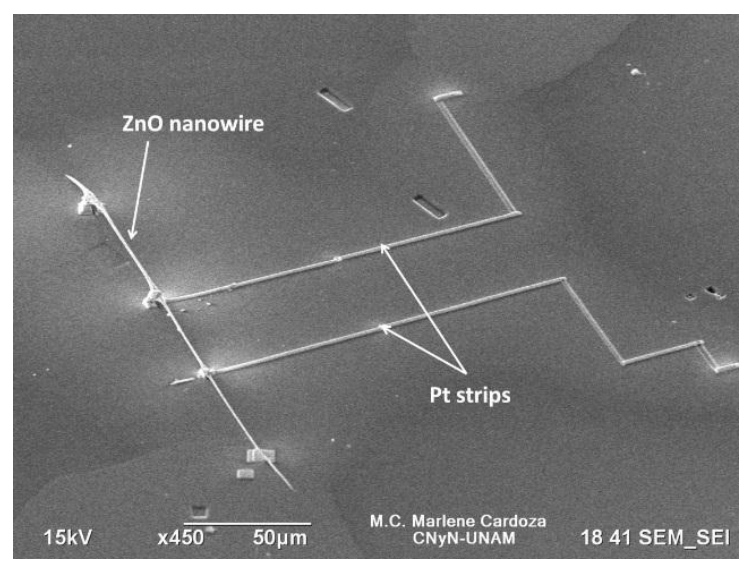

Figure 1. SEM image of fabricated single $\mathrm{ZnO}$ nanowire gas sensor device ( $\left.\mathrm{M}_{1}\right)$.

Figure 2 shows the characteristic I-V curves measured for each sensor with an ohmic behavior clearly evident in the current range of -50 to $+50 \mathrm{nA}$. As we can appreciate, there is a strong dependence on the nanowire diameter in the I-V characteristics. Since nanowires have a cylindrical shape, their electrical resistance can be estimated by Equation (2).

$$
R=\rho \frac{4 l}{\pi D^{2}}=\frac{1}{n e \mu} \frac{4 l}{\pi D^{2}}
$$

Where $R$ is the nanowire electrical resistance, $\rho$ is the nanowire electrical resistivity, $l$ is the inner electrode distance, $\mu$ is the electronic mobility, and $D$ is the nanowire diameter. According to the above equation, electrical resistance of the nanowires should have a major value as the diameter decreases. Nevertheless, this behavior would correspond to a passive electrical resistance where no changes in its electron density (n) are expected. Electrical resistances of single $\mathrm{ZnO}$ nanowires obtained from I-V curves shown in Figure 2 were $2.05 \times 10^{5} \Omega, 3.58 \times 10^{4} \Omega$, and $4.38 \times 10^{3} \Omega$ for $\mathrm{M}_{1}$, $\mathrm{M}_{2}$, and $\mathrm{M}_{3}$, respectively. Considering that $\mathrm{I}-\mathrm{V}$ measures were performed at room conditions, the $\mathrm{ZnO}$ nanowires have trapped charges on their surface causing electrical resistance increments. Then $\mathrm{ZnO}$ electrical resistance turns out to be active and dependent on the electron density. This effect can be more evident as the diameter of the nanowire reduces and the resistance is more affected by surface phenomena.

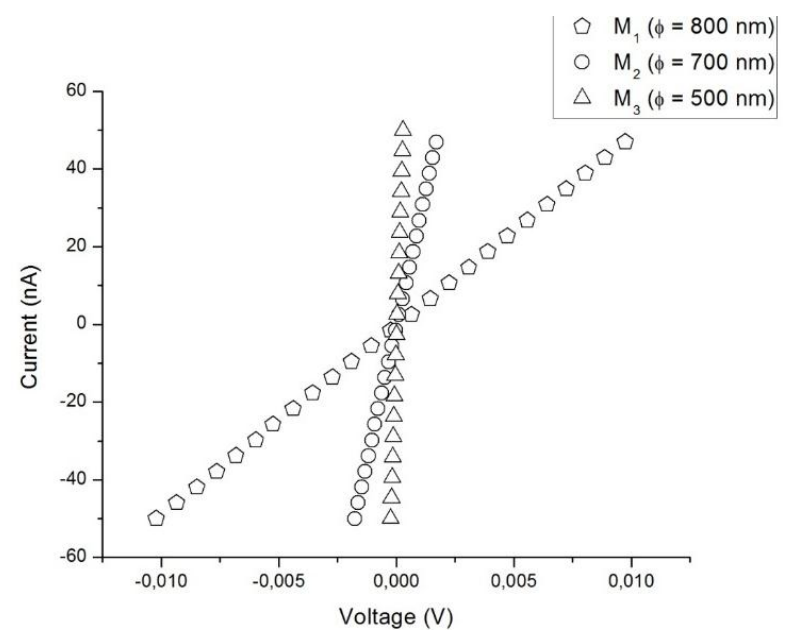

Figure 2. I-V characteristics of single $\mathrm{ZnO}$ nanowire devices showing an ohmic behavior. 
Figure 3 consists of a scheme showing the proposal detection mechanism for a $100 \mathrm{~s} \mathrm{H}_{2}$ pulse at a $121 \mathrm{ppm}$ concentration in the $\mathrm{M}_{2}$ device at $373.15 \mathrm{~K}$. In the first stage, the $\mathrm{ZnO}$ nanowire has oxygen ions trapped on its surface; then, since $\mathrm{ZnO}$ is an n-type semiconductor, the above causes the appearance of a charge depletion region next to the nanowire surface. $\mathrm{ZnO}$ nanowire has nanoscale dimensions and a limited conduction channel, and it can be assumed that a large amount of its carriers are trapped in surface states. In the second stage, a pulse with a concentration of $121 \mathrm{ppm}$ of $\mathrm{H}_{2}$ was introduced into the homemade gas chamber. Hydrogen is very reactive with the oxygen ions adsorbed on the semiconductor nanowire surface, becoming vapor water as a product (normally carried out by the dry air $\mathrm{H}_{2}$ flow), and is consequently released back to the surface-trapped electrons. This interaction results in an increment of the majority charge carriers in the nanowire and, hence, a decrease from 36.3 to $31.8 \mathrm{k} \Omega$ of the nanowire resistance in a time of $27 \mathrm{~s}$. This demonstrates that even a low concentration $\mathrm{H}_{2}$ pulse of $121 \mathrm{ppm}$ can induce a measurable change in the nanowire resistance. In the last stage, the depletion region is rebuilt when the $\mathrm{H}_{2}$ gas pulse is removed, maintaining only the gas reference and leading again to gradual nanowire surface oxidation (until the nanowire resistance reaches at least $0.9 \mathrm{R}_{\mathrm{a}}$ ) and getting a recuperation time of $28 \mathrm{~s}$.

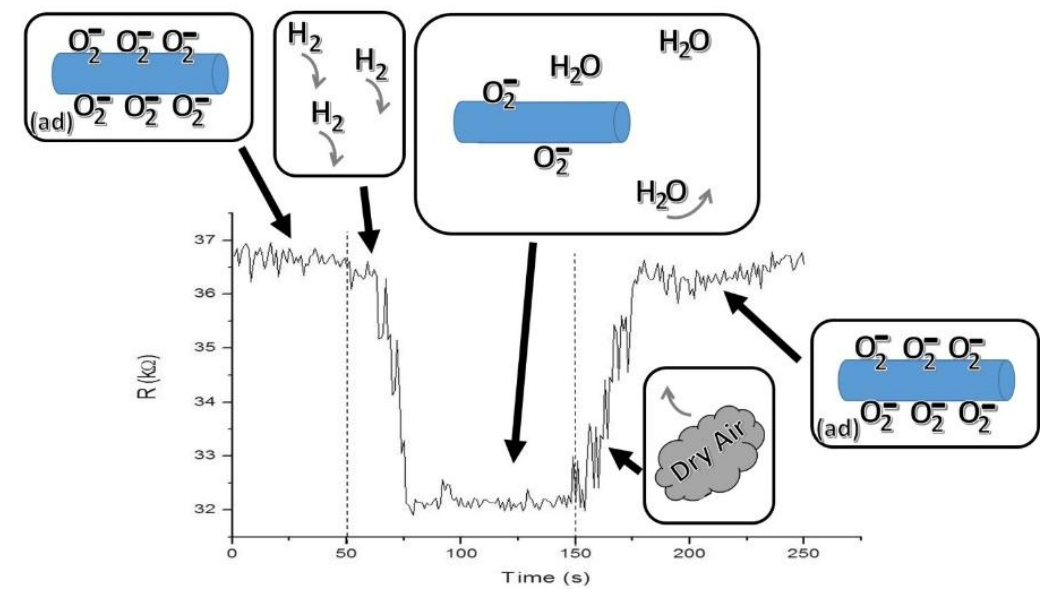

Figure 3. Sensing mechanism scheme occurring in one of the semiconductor devices, through the resistance change of $\mathrm{M}_{2}$ at a pulse concentration of $121 \mathrm{ppm}$ of $\mathrm{H}_{2}$ at $373.15 \mathrm{~K}$.

Figure 4a,b show the $\mathrm{ZnO}$ nanowire resistance variations for the different $\mathrm{H}_{2}$ pulses at room temperature and at $373.15 \mathrm{~K}$, respectively. It is observed at both temperatures that, at the lowest $\mathrm{H}_{2}$ concentration tested $(121 \mathrm{ppm})$, the calculated $\% \Delta \mathrm{R}$ is greater for the $\mathrm{ZnO}$ nanowire of smaller diameter $\left(\mathrm{M}_{3}\right)$, thus impacting the higher level of detection of the sensor element. This result is consistent considering that the smaller the diameter, the smaller the conduction channel of the nanowire, and hence, the greater the influence of the electrons released back from its surface on the resistance of the nanowire. Moreover, it is also noted that the major change of resistance was at the lowest concentration for the three devices; meanwhile, at high concentrations ( $>417 \mathrm{ppm}$ ), increments of $\% \Delta \mathrm{R}$ of the nanowires are minimal (0.5\%-2.5\%). This shows that $\mathrm{ZnO}$ nanowires can detect very low concentrations of $\mathrm{H}_{2}$ and it is likely that the nanowire surface is getting depleted of oxygen at elevated concentrations of $\mathrm{H}_{2}$.

Finally, all gas sensor devices show higher $\% \Delta \mathrm{R}$ at $373.15 \mathrm{~K}$, obtaining even a significant resistance variation of the major nanowire diameter device $\left(\mathrm{M}_{1}\right)$ in response to a low $\mathrm{H}_{2}$ concentration pulse of $121 \mathrm{ppm}$. It is also demonstrated that the resistance variation at $373.15 \mathrm{~K}$ (Figure $4 \mathrm{~b}$ ) has a more stepped shape than at $300.15 \mathrm{~K}$ (Figure $4 \mathrm{a}$ ), showing a greater uniformity of resistance variation percentage values between the fabricated devices. 


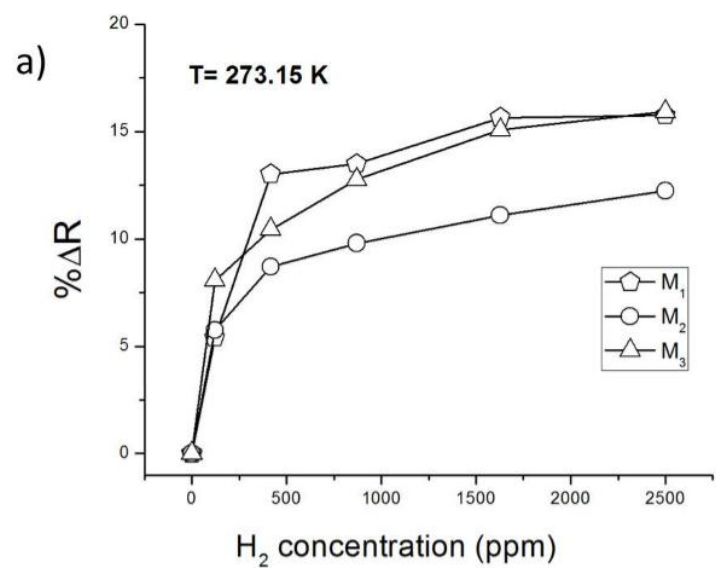

(a)

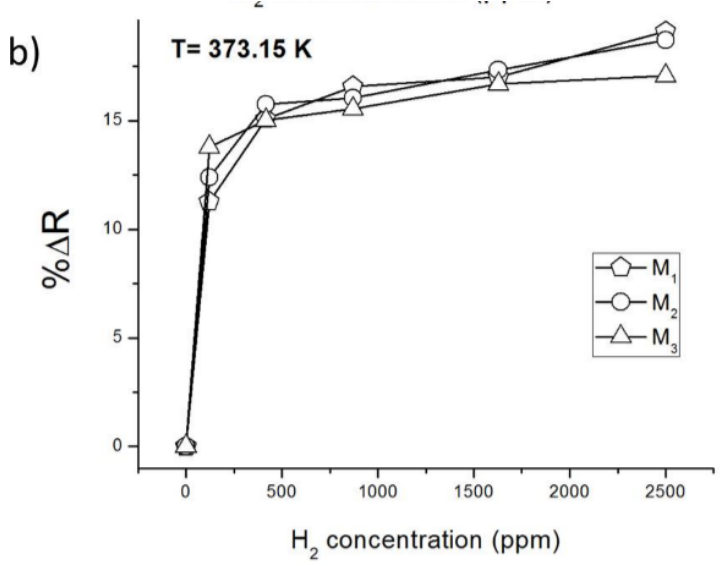

(b)

Figure 4. $\mathrm{ZnO}$ nanowire gas sensor devices percentage resistance changes for different $\mathrm{H}_{2}$ concentrations pulses. (a) At room temperature; (b) at $373.15 \mathrm{~K}$.

\section{Conclusions}

In summary, we have shown that single $\mathrm{ZnO}$ nanowires-based $\mathrm{H}_{2}$ sensors are an alternative for detecting low concentrations of $\mathrm{H}_{2}$ due to their limited conduction channel that allows even a low concentration of injected electrons to produce a measurable change in their resistance.

Acknowledgments: This work was supported by CONACYT-México Nos. 265700 and 253280 project. The authors also acknowledge Eduardo Murillo, Israel Gradilla and David Dominguez for their technical support.

Author Contributions: Luis A. Rios contributed with the photolithographic process and design of the aluminum probe required for the device fabrication. Rafael García-Gutíerrez carried out the $\mathrm{ZnO}$ nanowire synthesis. T. A. Zepeda fabricated the gas flow mixing gas system as well as discussion. José M. Romo-Herrera contributed mainly with discussion about the sensing mechanism at low $\mathrm{H}_{2}$ concentrations. Marlene N. Cardoza-Contreras is the principal author of this research article, and conducted fabrication of the single $\mathrm{ZnO}$ nanowire gas sensor devices, $\mathrm{I}-\mathrm{V}$ characterization, and carried out the gas sensor response experiments. All founding, contribution, discussions and guidance necessary to achieve the present work were supported by Oscar E. Contreras, group lead and Marlene N. Cardoza-Contreras.

Conflicts of Interest: The authors declare no conflict of interest.

\section{References}

1. Rai, P.; Song, H.M.; Kim, Y.S.; Song, M.K.; Oh, P.R.; Yoon, J.M.; Yu, Y.T. Microwave assisted hydrothermal synthesis of single crystalline $\mathrm{ZnO}$ nanorods for gas sensor application. Mater. Lett. 2012, 68, 90-93.

2. Chaia, G.Y.; Lupan, O.; Rusu, E.V.; Stratanc, G.I.; Ursaki, V.V.; Sontea, V.; Khallaf, H.; Chow, L. Functionalized individual $\mathrm{ZnO}$ microwire for natural gas detection. Sens. Actuators A 2012, 176, 64-71.

3. Kang, B.S.; Wang, H.T.; Rena,F.; Pearton, S.J.; Morey, T.E.; Dennis, D.M.; Johnson, J.W.; Rajagopal, P.; Roberts, J.C.; Piner, E.L.; et al. Enzymatic glucose detection using $\mathrm{ZnO}$ nanorods on the gate region of AlGaN/GaN high electron mobility transistors. Appl. Phys. Lett. 2007, 91, doi:10.1063/1.2825574.

4. Willander, M.; Nur, O.; Zhao, Q.X.; Yang, L.L.; Lorenz, M.; Cao, B.Q.; Zúñiga, P.J.; Czekalla, C.; Zimmermann, G.; Grundmann, M.; et al. Zinc oxide nanorod based photonic devices: Recent progress in growth, light emitting diodes and lasers. Nanotechnology 2009, 20, doi:10.1088/0957-4484/20/33/332001.

5. Dai, J.; Xu, C.; Xu, X.; Guo, J.; Li, J.; Zhu, G.; Lin, Y. Single ZnO microrod ultraviolet photodetector with high photocurrent gain. Appl. Mater. Interfaces 2013, 5, 9344-9348.

6. Ramgir, N.S.; Yang, Y.; Zacharias, M. Nanowire-Based Sensors. Small 2010, 6, 1705-1722.

7. Tien, L.C.; Sadik, P.W.; Norton, D.P.; Voss, L.F.; Pearton, S.J.; Wang, H.T.; Kang, B.S.; Ren, F.; Jun, J.; Lin, J. Hydrogen sensing at room temperature with Pt-coated $\mathrm{ZnO}$ thin films and nanorods. Appl. Phys. Lett. 2005, 87, doi:10.1063/1.2136070.

8. Tien, L.C.; Norton, D.P.; Gila, B.P.; Pearton, S.J.; Wang, H.-T.; Kang, B.S.; Ren, F. Detection of hydrogen with $\mathrm{SnO}_{2}$-coated $\mathrm{ZnO}$ nanorods. Appl. Surf. Sci. 2007, 253, 4748-4752. 
9. Ranwa, S.; Kumar, M.; Singh, J.; Fanetti, M.; Kumar, M. Schottky-contacted vertically self-aligned ZnO nanorods for hydrogen gas nanosensor application. J. Appl. Phys. 2015, 118, doi:10.1063/1.4926953.

10. García-Gutiérrez, R.; Barboza-Flores, M.; Berman-Mendoza, D.; Rangel-Segura, R.; Contreras-López, O.E. Luminescence and Structure of ZnO Grown by Physical Vapor Deposition. Adv. Mater. Sci. Eng. 2012, 2012, doi:10.1155/2012/872597.

(C) 2015 by the authors; licensee MDPI, Basel, Switzerland. This article is an open access article distributed under the terms and conditions of the Creative Commons by Attribution (CC-BY) license (http://creativecommons.org/licenses/by/4.0/). 